\title{
Identificação do Helicobacter pylori pela citologia do escovado gástrico: comparação com o método histológico
}

\author{
Helicobacter pylori identification by brush gastric cytology: \\ a comparison with histologic method
}

\author{
Ronaldo de Oliveira Custódio', Roberto Ruhman Daher², Yara R. Ximenes ${ }^{3}$, \\ Américo de Oliveira Silvério ${ }^{4}$ e Natália Ribeiro de O. Custódio ${ }^{5}$
}

\begin{abstract}
RESUM0
A proposta deste estudo foi a de verificar o valor da citologia do escovado gástrico no diagnóstico da infecção pelo Helicobacter pylori em pacientes submetidos à endoscopia digestiva, comparando-o a outro método endoscópico - a histologia. As endoscopias foram realizadas em 157 pacientes dispépticos, divididos em dois grupos: grupo $\mathrm{A}(\mathrm{n}=27)$ com úlcera duodenal, $\mathrm{B}(\mathrm{n}=130)$, sem úlcera. № grupo A, a porcentagem de pacientes positivos na citologia $(77,8 \%)$ foi similar à histologia $(74,1 \% ; \mathrm{p}=0,3)$. No grupo B, a citologia (71,5\%) foi superior à histologia (63,1\%; $\mathrm{p}=0,00002)$. A citologia do escovado gástrico é um método simples e prático. Foi eficiente para identificar a infecção pelo Helicobacter pylori em todos grupos de estudo.
\end{abstract}

Palavras-chaves: Identificação do Helicobacter pylori. Citologia. Histologia.

\begin{abstract}
The purpose of this study was to verify the efficacy of brush gastric cytology for diagnosis of Helicobacter pylori infection in patients, submitted to elective gastroscopy, compared to the method of endoscopic histology. Endoscopy was performed on 157 patients, divided into two dyspeptic groups: group $A(n=27)$ with duodenal ulcer; and group $B(n=130)$ without ulcer. In group A, the percentage of positive cases detected by brush cytology $(77.8 \%)$ was similar to histology $(74.1 \% ; p=$ $0.3)$. While in group $B$, brush cytology $(71.5 \%)$ was superior to histology $(63.1 \% ; p=0.00002)$. Brush cytology is a simple and useful diagnostic method. It was efficient for identification of Helicobacter pylori infection in both groups.
\end{abstract}

Key-words: Helicobacter pylori identification. Brush cytology. Histology.

Desde a descrição pioneira ate 0 surgimento de evidências do Helicobacter pylori ser o principal agente etiológico da doença ulcerosa duodenal ${ }^{1718}$, seu diagnóstico e tratamento tiveram uma dramática alteração. A doença ulcerosa duodenal é agora abordada como uma doença infecciosa, na qual a eliminação do agente causador cura esta condiçã $0^{26}$. A infecção está associada com 0 aumento de risco de carcinoma gástrico ${ }^{21}$, sendo esta bactéria tida como carcinógeno do tipo ${ }^{16}$. Também há acentuada associação entre a infecção crônica pelo Helicobacter pylori e o linfoma Malt ${ }^{7011}$.

Os testes diagnósticos são classificados em não-invasivos quando a endoscopia não é requerida e são baseados em evidências indiretas da presença da bactéria; invasivos ou endoscópicos quando detectam a infecção através de material obtido durante 0 curso de uma endoscopia (Tabela 1).

A citologia do esfregaço gástrico é um método já existente, porém pouco divulgado. 0 material é obtido por um escovado da mucosa gástrica com o qual se faz um esfregaço na lâmina de vidro. Após corada a lâmina, procede-se a pesquisa direta do Helicobacter pylori. É um método mais simples e mais rápido que o histopatológico, além de ser menos agressivo à mucosa gástrica por não se utilizar de biópsia. Há referências de que a sensibilidade da citologia é semelhante ou superior ao da histologia no diagnóstico da infecção pelo Helicobacter pylori ${ }^{3615} 19$.

\footnotetext{
1. Serviço de Gastroenterologia e Hepatologia do Hospital das Clínicas da Universidade Federal de Goiás, Goiânia, GO. 2. Departamento de Medicina de Medicina Tropical do Instituto de Patologia Tropical e Saúde Pública da Universidade Federal de Goiás, Goiânia, GO. 3. Laboratório CAPC, Goiânia, GO. 4. Serviço de Gastroenterologia do Hospital Geral de Goiânia, Goiânia, GO. 5. Curso de Medicina da Faculdade de Medicina da Universidade Federal de Goiás, Goiânia, GO. Endereço para correspondência: Dr. Ronaldo de Oliveira Custódio. Rua 9-A esq. c/ 16-A, no 344, setor Aeroporto, 74075-040 Goiânia, G0.

Tel.: 5562 229-1732, fax: 55622250952

e-mail: rocust@ig.com.br

Recebido para publicação em 16/8/2004

Aceito em 3/5/2005
} 
Tabela 1 - Testes diagnósticos utilizáveis para a detecção da infecção por Helicobacter pylori.

\begin{tabular}{lll}
\hline Testes não invasivos & \multicolumn{2}{c}{ Testes invasivos } \\
\cline { 2 - 3 } & biópsia & escovado gástrico \\
\hline Teste respiratório & histologia & citologia \\
Testes sorológicos & teste da urease & cultura \\
PCR em antígeno fecal & PCR & \\
& imunohistoquímica & \\
& cultura & \\
\hline
\end{tabular}

Modificado de: Libera e coll ${ }^{15}$; Hung e cols ${ }^{9}$; Leodolter e cols ${ }^{14}$; Suerbauem \& Michetti2 ${ }^{26}$; Drum e cols ${ }^{5}$; Howden e cols ${ }^{6}$.

0 objetivo é realizar a identificação morfológica do Helicobacter pylori mediante a citologia do escovado gástrico, divulgar o método e comparar sua acurácia com a histologia.

\section{PACIENTES E MÉTODOS}

Estudo prospectivo, comparativo e aberto. Foram incluídos no estudo 157 pacientes com dispepsia, que seriam submetidos por indicação prévia de outros profissionais dos ambulatórios do Hospital das Clínicas (HC) da Universidade Federal de Goiás (UFG) à endoscopia digestiva. Estes correspondem a 93 mulheres e 64 homens (Tabela 2), com média de idade de 41,8 \pm 15,6 anos. 0 período de duração do estudo foi de setembro de 2002 a junho de 2003. Todos eles assinaram 0 termo de consentimento livre e esclarecido

Tabela 2 - características da população estudada e sua relação com os grupos de estudo.

\begin{tabular}{lccccc}
\hline Sexo & Número & Percentagem & Idade ( \pm dp) & Grupo A & Grupo B \\
\hline Homens & 64 & 40,8 & $44,0 \pm 16,8$ & 18 & 46 \\
Mulheres & 93 & 59,2 & $40,0 \pm 14,5$ & 9 & 84 \\
\hline Total & 157 & 100,0 & $\mathrm{p}=0,08$ & 27 & 130 \\
\hline
\end{tabular}

Grupo A = dispépticos ulcerosos duodenais;

Grupo B = dispépticos não-ulcerosos.

e 0 estudo foi realizado com aprovação do comitê de ética do HC/UFG. Foram excluídos pacientes com neoplasia gástrica, úlcera gástrica, hemorragia digestiva alta e pacientes submetidos a gastrectomia. De acordo com achados endoscópicos, os pacientes foram agrupados em: grupo A, 27 pacientes dispépticos ulcerosos duodenais; grupo B, 130 pacientes dispépticos nãoulcerosos ( Tabela 3). Os achados endoscópicos no esôfago não foram considerados na formação dos grupos. 0 material era colhido obedecendo à seguinte ordem: escovação e biópsia. 0 instrumental consistia de escova de citologia endoscópica e pinças de biópsia reutilizáveis. Foi utilizada escova da marca MTW embutida em cateter de teflon com esfera de metal na

Tabela 3 - Análise da distribuição dos 157 pacientes segundo grupos de estudo e positividade nos dois métodos empregados.

\begin{tabular}{lrrrrr}
\hline & \multicolumn{2}{c}{ Citologia a } & & \multicolumn{2}{c}{ Histologia b } \\
\cline { 2 - 3 } & $\mathrm{n}^{0}$ & $\%$ & & $\mathrm{n}^{0}$ & $\%$ \\
\hline Grupo $\mathrm{A}(\mathrm{n}=27)$ & 21 & 77,8 & & 20 & 74,1 \\
Grupo $\mathrm{B}(\mathrm{n}=130)$ & 93 & 71,5 & & 82 & 63,1 \\
\hline Total $(\mathrm{n}=157)$ & 114 & 72,6 & & 102 & 65,1
\end{tabular}

Grupo A: a x b p =0,3* Grupo B: a x b p =0,00002*

(*Qui-quadrado com correção de Yates). extremidade; sua montagem e desmontagem é feita pela extremidade distal.

A mucosa do corpo e antro foram escovadas, e em seguida a escova era friccionada em duas lâminas de vidro as quais eram borrifadas com fixador a base de álcool, secadas, colocadas em um cilindro de plástico, vedado e encaminhado ao laboratório para coloração pelo método de Giemsa. Para a histologia, duas amostras do antro e duas do corpo eram colhidas e colocadas em recipiente contendo formalina a dez por cento e encaminhado para exame histopatológico e, posterior coloração pelos métodos de Giemsa e HE. A esterilização da escova de citologia e a pinça de biópsia é padronizada no serviço utilizando o glutaraldeído a 2\% por 8 horas $^{24}$. As lâminas de citologia foram examinadas por um citologista e os exames de histopatologia por outro patologista, os quais não tinham informações entre si. Considerando padrão ouro quando era visto microrganismo na histopatologia.

Na comparação das frequiências obtidas nos dois métodos foram empregados o qui-quadrado com correção de Yates e 0 exato de Fisher quando necessário. Foram considerados níveis de significância estatística os valores de pd<0,05.

\section{RESULTAD0S}

Nos 157 pacientes incluídos no estudo, não houve diferença nas médias de idade entre homens e mulheres (44,0 $\pm 16,8$ versus 40,0 $\pm 14,5 ; p=0,08)$, demonstradas na Tabela 2. Nestes, foram pesquisados a presença da bactéria Helicobacter pylori pelos métodos citológico e histológico com os seguintes achados: $114(72,6 \%)$ positivos pela citologia e $102(65,1 \%)$ positivos pela histologia ( Tabela 3); 129 ( $82,2 \%)$ positivos pela associação dos métodos, citologia e histologia ( Tabela 4).

Tabela 4 - Demonstração da positividade da infecção nos grupos A e B, considerando a associação da citologia e histologia.

\begin{tabular}{lrrrrr}
\hline & \multicolumn{2}{c}{ Positivos } & & \multicolumn{2}{c}{ Negativos } \\
\cline { 2 - 3 } & $\mathrm{n}^{0}$ & $\%$ & & $\mathrm{n}^{0}$ & $\%$ \\
\hline Grupo $\mathrm{A}(\mathrm{n}=27)$ & 24 & 88,9 & & 3 & 11,1 \\
Grupo $\mathrm{B}(\mathrm{n}=130)$ & 105 & 80,8 & & 25 & 19,2 \\
\hline Total & 129 & 82,2 & & 28 & 17,8 \\
\hline
\end{tabular}

$\mathrm{P}=0,24 \quad \mathrm{RR}=1,74(0,565,37) \quad \mathrm{OR}=1,90(0,51$ 10,62) (Exato de Fisher)

Na Tabela 3, os resultados são comparados nos dois grupos de pacientes. A citologia foi similar à histologia em pacientes com dispepsia ulcerosa duodenal ( grupo A, 77,8\% versus $74,1 \%$, $\mathrm{p}=0,3$ ) , e superior à histologia em pacientes com dispepsia nãoulcerosa (grupo $B, 71,5 \%$ versus $63,1 \%, p=0,00002$ ).

ATabela 4 sugere que os resultados da frequiência da infecção pelo Helicobacter pylori utilizando-se os dois métodos ( citologia e/ou histologia) são semelhantes entre os dois grupos estudados (grupo A, 88,9\% versus grupo B, 80,8\%, $\mathrm{p}=0,24$ ).

\section{DISCUSSÃ0}

Os métodos mais utilizados na identificação do Helicobacter pylori ainda são os invasivos que se utilizam da endoscopia 
digestiva. 0 mais usual destes é 0 método histológico, que tem com vantagem adicionar dados importantes sobre 0 estado da mucosa gástrica, como 0 grau de inflamação, atividade inflamatória, atrofia da mucosa, identificação de metaplasia intestinal na mucosa gástrica e outras informações úteis no cuidado ao paciente. Porém, é um método relativamente caro, demorado e a acurácia na identificação da bactéria sofre influências na dependência do número de fragmentos de biópsia ${ }^{120}$.

0 método histológico deve ser realizado com a obtenção de dois ou mais fragmentos da mucosa antral. Há melhora da acurácia se colhido dois fragmentos antral e dois do corpo gástric ${ }^{20}$. A sensibilidade da histologia na identificação do Helicobacter pylori, quando usadas as colorações HE e Giemsa, pode ser baixa se a densidade de bactérias é pequena e boa se a densidade é alta. Laine e coll ${ }^{13}$ encontrou variação da sensibilidade de acordo com a densidade bacteriana para $0 \mathrm{HE}$ de $70 \%$ a $98 \%$ com especificidade variando de $89 \%$ a $98 \%$, e para 0 Giemsa, sensibilidade de $64 \%$ a $96 \%$ e especificidade de $98 \%$ a $100 \%$.

0 exame citológico é um método descrito há uma década ${ }^{319}$, porém ainda é pouco estudado. Verificamos sua acurácia na identificação do Helicobacter pylori como mais um método a ser associado aos já convencionais, comparando-0 com a histopatologia num conjunto de 157 pacientes que foram submetidos à endoscopia digestiva alta por causa das queixas de dispepsia.

Para a proposta deste trabalho, os pacientes foram divididos em dois grupos de estudo:

Grupo A: pacientes dispépticos com úlcera duodenal. Grupo B: pacientes dispépticos sem úlcera. 0 sistema de classificação de distúrbios funcionais gastrintestinais "Roma II" não recomenda 0 uso do termo dispepsia não-ulcerosa com 0 significado de dispepsia funcional ${ }^{4}$. A dispepsia funcional é um diagnóstico de exclusão, além da endoscopia seriam necessárias outras avaliações para a sua confirmação, portanto não podemos chamar 0 grupo B de dispepsia funcional.

Considerando os 157 pacientes estudados encontramos: 114 (72,6\%) positivos pelo método citológico, 102 (65\%) positivos pelo método histológico (Tabela 3) e 129 (82,2\%) positivos por ambos os métodos, citologia e/ou histologia (Tabela 4). Este alto índice de infecção pode ser devido à população selecionada de pacientes sintomáticos bem como o seu baixo nível socio-econômico.

A sensibilidade da citologia calculada frente a histologia foi de 85,3\%. Cerca de 27 casos positivos pelo método citológico foram negativos pela histologia: a escova de citologia abrange uma área maior a ser estudada e estes dados sugerem ser a citologia mais sensível que os métodos que se utilizam de biópsia na identificação do Helicobacter pylori. 0 microrganismo está distribuído irregularmente no muco gástrico na superfície epitelial, e a relativa baixa densidade da bactéria em vários grupos de pacientes pode levar a resultados falso-negativos em métodos de biópsia ${ }^{12}$. A comparação de um método que se utiliza do esfregaço obtido por um escovado gástrico - a citologia, com um método que se utiliza de biópsia - a histologia, influenciou na especificidade do primeiro que foi de 50,9\%. Talvez os achados justifiquem ser a citologia um método com mais sensibilidade que a histologia, sendo necessárias pesquisas comparativas de ambos com métodos mais sensíveis, ou com a combinação de dois ou três métodos, para determinar a sua real sensibilidade e especificidade.

Năo houve diferença entre a frequêencia da infecção por Helicobacter pylori no grupo A $(88,9 \%)$ versus grupo B $(80,8 \%) ; p=0,24$ (Tabela 4). No processo de ulcerogênese, as pesquisas apontam para as cepas ulcerogênicas ${ }^{22}$ associadas a características próprias do hospedeiro, as quais não foram objetos deste estudo.

Nos pacientes do grupo $\mathrm{A}$ ( pacientes dispépticos com úlcera duodenal) , a citologia foi similar à histologia $(77,8 \% \mathrm{X} 74,1 \%$, $\mathrm{p}=0,3$ ) na identificação do Helicobacter pylori (Tabela 3) . No grupo $B$ ( pacientes dispépticos sem úlcera), a citologia ( $71,5 \%)$ foi superior à histologia (63,1\%; p=0,00002) (Tabela 3) .

Os resultados quando comparados com trabalhos de outros autores que destacam ser 0 método fácil de ser executado, mais rápido que a histologia, e a sensibilidade é igual ou superior aos métodos que se utilizam de biópsia, foram concordantes. Estes resultados deixam claro que a citologia do escovado gástrico é um método simples e útil na identificação da infecção pelo Helicobacter pylori, nas diferentes formas de dispepsia. É uma opção para ser associado a outros métodos na tentativa de aumentar a acurácia do diagnóstico da infecção por Helicobacter pylori. Poderia ser considerado para aqueles pacientes que têm a biópsia gástrica endocópica contra-indicada.

\section{AGRADECIMENTOS}

Desejo expressar o meu apreço às pessoas mencionadas por suas importantes contribuiç̃̃es: Prof. Dr. Décio Chinzon, Prof. Dr. Heitor Rosa e Prof. Dr. Maurício S. Brasil Leite.

\section{REFERÊNCIAS BIBLIOGRÁFICAS}

1. Atalay C, Atalay G, Altinok M. Serum Helicobacter pylori IgG and IgA levels in patients with gastric cancer. Neoplasma 50: 185-190, 2003.

2. Bazzoli F. Key points from the revised Maastrich Consensus Report: the impact on general practice. European Journal of Gastroenterology \& Hepatology 13: S3-S7, 2001.

3. De Francesco F, Nicotina PA, Picciotto M, Martines F, Ferlazzo G, D'Aquino A. Helicobacter pylori in gastroduodenal diseases: rapid identification by endoscopic brush cytology. Diagnostic Cytopathology 9: 430-433, 1993.

4. Drossman DA, Corazziari E, Talley NJ, Thompson WG, Whitehead WE. Functional dyspepsia. The functional gastrointestinal disorders. Second edition. Library of Congress 302-350, 2000.

5. Drum B, Koleztko S, Oderda G. Helicobacter pylori infection in children: consensus statement. Journal of Pediatric Gastroenterology and Nutrition 30: 207-213, 2000.

6. Faraker CA. Diagnosis of Helicobacter pylori in gastric brush and biopsy specimens stained by Romanowsky and immunocytochemical methods: comparison with the ClOtest. Cytopathology 7: 108-119, 1996.

7. Genta RM, Hammer HW, Graham DY. Gastric lymphoid follicles in Helicobacter pylori infection: frequency, distribution, and response to triple therapy. Human Pathology 24: 577-583, 1993. 
8. Howden CW, Hunt RH. Guidelines for the management of Helicobacter pylori infection. The American Journal of Gastroenterology 93: 2330-2338,1998.

9. Hung CT, Leung WK, Chan FK, Sung JJ. Comparison of two new rapid tests for diagnosis of Helicobacter pylori infection in Chinese patients. Digestive and Liver Diseases 34: 111-115, 2002.

10. Isaacson $P G$, Spencer J. Is gastric lymphoma an infectious diseases? Human Pathology 24: 569-570, 1993.

11. Isaacson PG. Gastrointestinal lymphoma. Human Pathology 24: 1020-1029, 1994.

12. Khulusi S, Mendall MA, Patal P, Levy J, Badve S, Badve S, Northfield TC. Helicobacter pylori infection density and gastric inflammation in duodenal ulcer and non-ulcer subjects. Gut 37:319-324,1995.

13. Laine L, Lewin DN, Naritoku W, Cohen H. Prospective comparison of H\&E, Giemsa, and Genta stains for the diagnosis of Helicobacter pylori. Gastrointestinal Endoscopy 45: 463-467, 1997.

14. Leodolter A, Vaira D, Bazzoli F, Schutze K, Hirschl A, Megraud F, Malfertheiner P. European multicentre validation trial of two new noninvasive tests for the detection of Helicobacter pylori antibodies: urine based ELISA and rapid urine test. Aliment Pharmacology 18: 927-931, 2001.

15. Libera MD, Pazzi P, Carli G, Contato E, Piva I, Scagliorini R, Merighi A, Ricci N, Gullini S. Brush cytology: A reliable method to detect Helicobacter pylori. Journal of Clinical Gastroenterology 22: 317-321, 1996.

16. Logan RPH. Helicobacter pylori and gastric cancer. Lancet 34: 1078-1079, 1994.

17. Marshall BJ, Warren JR. Unidentified curved bacilli in the stomach of patients with gastritis and peptic ulceration. Lancet 1311-1314, 1984.

18. Marshall BJ, Warren JR, Francis GJ, Langton SR, Godwin CS, Blincow ED. Rapid urease test in the management of Campylobacter pyloridis associated gastritis. The American Journal of Gastroenterology 82: 200210, 1987.

19. Mendoza ML, Martin-Rabadan P, Carrion I, Morillas JD, Lopez-Alonso G, Diaz-Rubio M. Helicobacter pylori infection. Rapid diagnosis with brush cytology. Acta Cytologica 37: 181-185, 1993.

20. Morris A, Ali MR, Brown P, Lane M, Patton K. Campylobacter pylori infection in biopsy specimens of gastric antrum: laboratory diagnosis and estimation of sampling error. Journal of Clinic Pathology 42: 727-732, 1989.

21. Nomura A, Stemmerman G, Chyon PH, Kato I, Perez-Perez GI, Blaser MJ. Helicobacter pylori infection and gastric carcinoma amongJapanese Americans in Hawaii. The New England Journal of Medicine 325: 1132-1136 1991.

22. Regula J, Hennig E, Burzykoswski T, Orlowska J, Przytulski K, Polkow M, Dziurkowska-Marek A, Marek T, Nowak A, Butruk E, Ostrowski J. Multivariate analysis of risk factors for development of duodenal ulcers in Helicobacter pylori-infected patients. Digestion 67: 25-31, 2003.

23. Schnading VJ, Bigio EH, Gourley WK, Stewart GD, Newton GA, Shabot JM. Identification of Campylobacter pylori by endoscopic brush cytology. Diagnostic Cytopathology 6: 227-234, 1990.

24. Silva AB, Favaro BL, Araujo MB, Passos MLP, Toledo SV. Manual de Padronização de Procedimentos de Enfermagem do Hospital das Clínicas da Universidade Federal de Goiás, Goiânia, 2003.

25. Sjunnesson H, Falt T, Sturegard E, Abu-Al Soud W, Ljungh A, Wadstrom T. PCRdenaturing gradient gel electrophoresis and two feces antigen tests for detection of Helicobacter pylori in mice. Current Microbiology 47: 278-285, 2003.

26. Suerbaum S, Michett P. Helicobacter pylori infection. The New England Journal of Medicine 347: 1175-1176, 2002.

27. Tolentino MM, Faifer JG. Úlcera Péptica duodenal. In: SOBED (ed) Sociedade Brasileira de Endoscopia digestiva, Editora Medsi, Rio de Janeiro, p. 177-215, 1994. 